\title{
Traduire
}

Revue française de la traduction

$236 \mid 2017$

Traducteurs en blouse blanche

\section{Traduire la microstoria}

\section{Sandro Landi}

\section{(2) OpenEdition \\ Journals}

Édition électronique

URL : http://journals.openedition.org/traduire/921

DOI : 10.4000/traduire.921

ISSN : 2272-9992

Éditeur

Société française des traducteurs

Édition imprimée

Date de publication : 1 juin 2017

Pagination : 71-74

ISSN : 0395-773X

Référence électronique

Sandro Landi, «Traduire la microstoria », Traduire [En ligne], 236 | 2017, mis en ligne le 01 juin 2019, consulté le 29 juin 2019. URL : http://journals.openedition.org/traduire/921 ; DOI : 10.4000/

traduire.921 


\section{Traduire la microstoria}

\section{Sandro Landi}

Selon le témoignage de Jacques Revel, " le terme de micro-histoire (microstoria) est entré il y a une trentaine d'années dans le vocabulaire de l'historiographie(1) ". Ces quelques mots permettent de situer un moment clé : au début des années 1980, la " micro-histoire ", indiquant traditionnellement la petite histoire (" de la micro-histoire tout au plus ", selon l'allusion ironique de Raymond Queneau dans Les Fleurs bleues)(2), commence à désigner, dans l'historiographie française, un projet nouveau, venu d'Italie. Deux traductions, presque simultanées, parues dans la revue Le Débat, dirigée par Pierre Nora, marquent ce tournant : "Signes, traces, pistes. Racines d'un paradigme de l'indice " de Carlo Ginzburg et "La micro-histoire ", par Carlo Ginzburg et Carlo Poni(3).

À propos de ces articles, il est possible de remarquer que si le décalage temporel qui les sépare de leur parution en Italie est court, le travail d'ajustement dont leurs titres font l'objet est, en revanche, important. Le premier est publié sous le titre : Spie : radici di un paradigma indiziario dans un ouvrage collectif, Crisi della Ragione, chez l'éditeur Einaudi en 1979(4). Spie (pluriel de spia) est un mot qui indique aussi bien l'espion que l'indice ou le symptôme(5). Cette ambiguïté sémantique, sans doute recherchée par l'auteur, est intraduisible et reste sans écho dans le titre français. Le second, dont le titre original est : Il nome e il come : scambio ineguale

(1) J. Revel, "Microstoria ", in Historiographies, I, Concepts et débats, sous la direction de C. Delacroix, F. Dosse, P. Garcia, N. Offenstadt, Paris, Gallimard, 2010, p. 529-534, à la p. 529. Sur Jacques Revel et la microhistoire, cf. F. Trivellato, "Microstoria/Microhistoire/Microhistory ", in La forza delle incertezze. Dialoghi storiografici con Jacques Revel, sous la direction de A. Romano e S. Sebastiani, Bologne (Italie), II Mulino, 2016, p. 49-69.

(2) R. Queneau, Les Fleurs bleues, Paris, Gallimard, 1965, p. 84-85.

(3) Le Débat, 1980/6 n 6, p. 3-44, traduction de Jean-Pierre Cottereau et Le Débat, 1981/10 n 17, p. 133-136, traducteur anonyme.

(4) Cet article a été traduit en 14 langues, cf. C. Ginzburg, Le fil et les traces. Vrai, faux, fictif, traduit de l'italien par M. Rueff, Lagrasse, Verdier, 2006, p. 514-515 ; l'édition française la plus récente est "Traces. Racines d'un paradigme indiciaire ", in C. Ginzburg, Mythes, emblèmes, traces. Morphologie et histoire, Nouvelle édition augmentée, traduit de l'italien par M. Aymard, C. Paoloni, E. Bonan, M. Sancini-Vignet, revue par M. Rueff, Lagrasse, Verdier, 2010, p. 218-294.

(5) http://www.treccani.it/vocabolario/spia/. 
e mercato storiografico, est à l'origine une communication présentée au colloque " Les Annales et l'historiographie italienne " (Rome, 1979), ensuite publiée, cette même année, dans la revue Quaderni storici(6). Ici le choix du traducteur anonyme est radical : en effet, au lieu de traduire "Le nom et la manière. Échange inégal et marché historiographique ", il crée un titre nouveau à partir d'un mot (microstoria) présent dans le texte italien.

II faut souligner qu'à cette époque les historiens italiens qui se réclament de la microstoria, à savoir d'un mode opératoire fondé sur la variation d'échelle, sur la mise en valeur de détails infimes, sur l'essai comme forme de l'enquête, n'utilisent ce terme que de façon occasionnelle. Comme le rappelle Carlo Ginzburg(7), le premier à employer le mot microstoria " de manière autonome " est Primo Levi dans // Sistema periodico (1975)(8) ; ce sera un cousin de l'écrivain, Giovanni Levi, qui l'introduira, en 1977, dans le champ historiographique(9). On remarquera également que dans l'article Spie (...), qui constitue pourtant une illustration éclatante de cette approche, le terme de microstoria n'est jamais employé. Le choix de traduction de la revue Le Débat témoigne donc de la volonté de nommer et de délimiter, pour le public français, un mouvement qui, à son commencement en Italie, ne se distingue pas par un nom ni par une identité strictement définis.

Par ailleurs, l'article "La micro-histoire " est très peu fidèle à son original. Comme le dit le sous-titre (scambio ineguale e mercato storiografico), ce qui en constitue l'objet est le " marché historiographique " et, plus précisément, l'" échange inégal " qui caractérise ce domaine des relations intellectuelles entre la France et l'Italie(10). Selon ses auteurs, l'article a valeur " pronostique "(11) : il prévoit l'épuisement du modèle de l'histoire quantitative prônée par la deuxième génération des Annales et l'avènement d'une " nouvelle tendance de la recherche ", fondée sur " une analyse extrêmement rapprochée " de phénomènes (la vie d'une communauté, d'un groupe, d'un individu ॥), dont le principal dénominateur est le nom propre, c'est-à-dire le plus individuel des indices(12). Cette approche historique, qualitative et " artisanale ", si elle se révèle

(6) Quaderni storici, 14, 1979, p. 181-189.

(7) C. Ginzburg, "Microhistoire : deux ou trois choses que je sais d'elle ", in Le fil et les traces, p. 361-405, à la p. 369.

(8) P. Levi, I/ sistema periodico, Turin, Einaudi, 1975, p. 229 : " II lettore, a questo punto, si sarà accorto da un pezzo che questo non è un trattato di chimica [...]. Ė, o avrebbe voluto essere, una microstoria, la storia di un mestiere e delle sue sconfitte, vittorie e miserie [...]"; "Arrivé à ce point, le lecteur se sera rendu compte depuis un moment qu'il ne s'agit pas ici d'un traité de chimie [...]. Mais il s'agit pourtant bien d'histoire en quelque sorte, ou plutôt on aurait voulu qu'il s'agisse d'une microhistoire, l'histoire d'un métier et de ses défaites, victoires et misères [...]. Traduction proposée par Ginzburg, "Microhistoire ", p. 369.

(9) Ginzburg, "Microhistoire ", p. 370.

(10) Ginzburg, Poni, " II nome e il come ", p. 181 : "Cominciamo da una constatazione banale. Nel corso di questo mezzo secolo gli scambi storiografici tra Italia e Francia sono stati fortemente squilibrati. L'Italia ha ricevuto molto più di quanto ha dato".

(11) Ibidem : "Il nostro sarà quindi un discorso più prognostico che diagnostico ".

(12) Ibidem, p. 186. 
comme la plus efficace pour exploiter la richesse documentaire des archives italiennes(13), est aussi en mesure de produire, à plus long terme, un rééquilibrage des rapports entre les deux traditions historiographiques(14). Ces considérations, vaguement tiers-mondistes, ne trouvent pas de place dans la version française qui s'intéresse surtout à la micro-histoire comme " science du vécu "(15), et à la manière dont elle peut concourir à l'étude des classes subalternes, suivant notamment l'exemple de l'anthropologie historique pratiquée, ces mêmes années, par Edward P. Thompson(16).

Quoique partielle et ciblée, cette traduction marque le début de l'acclimatation de la microstoria dans I'historiographie française. Le pronostic formulé par les historiens italiens semble en effet s'avérer, car deux ouvrages de Carlo Ginzburg font l'objet d'une traduction rapprochée : I Benandanti (Turin, Einaudi, 1966)(17) et le célèbre II formaggio e i vermi. I/ cosmo di un mugnaio del '500, (Turin, Einaudi, 1976)(18). Ce processus se révèle toutefois long et, surtout, comme le souligne Jacques Revel dans un premier bilan de l'expérience micro-historique en France, circonscrit à un milieu : la réception de la microstoria, écrit-il, " est restée concentrée à l'intérieur d'un nombre relativement restreint de groupes, d'institutions, de chantiers de recherche (dont la cartographie serait d'ailleurs intéressante à faire)(19) „. Si l'on essaye de développer ces observations, il est aisé d'affirmer que la traduction et la réception de la microstoria répondent aux dynamiques propres à la communauté des historiens des Annales, qui va de facto se constituer, dans les années 1980 et 1990, comme la principale communauté d'interprétation du mouvement micro-historique en France. Dans un contexte de remise en question des certitudes disciplinaires, l'éditorial " Histoire et sciences sociales. Un tournant critique ",

(13) Ibidem, p. 181-182 : "Abbiamo parlato di scambio ineguale e mercato storiografico. Ma paese dipendente non vuol dire necessariamente paese povero. La situazione di dipendenza storiografica dell'Italia si accompagna notoriamente a una straordinaria ricchezza di quel materiale documentario, senza il quale lo storico non può lavorare".

(14) Ibidem, p. 189 : "In questo senso è forse lecito prevedere, nei prossimi anni, uno scambio tra storiografia italiana e storiografia francese meno ineguale che in passato ".

(15) Ginzburg, Poni, "La micro-histoire ", p. 5.

(16) Ibidem, p. 4 : " Une prosopographie de la masse, donc, analogue à celle que proposait E.P. Thompson, et qui devrait déboucher sur une série d'études de cas, sans pourtant exclure, on l'a dit, les recherches sérielles". Sur la difficulté de traduire l'œuvre d'E.P. Thompson en contexte français, voir les considérations de J. Boutier et A. Virmani, "Présentation ", in E.P. Thompson, Les usages de la coutume. Traditions et résistances populaires en Angleterre XVII-XIXe siècles, traduit de l'Anglais par J. Boutier et A. Virmani, Paris, Gallimard/ Le Seuil, 2015, p. 34-39.

(17) Les Batailles Nocturnes. Sorcellerie et rituels agraires en Frioul, XVIe-XVIIe siècles, Verdier, 1980, traduit par l'ethnologue Giordana Charuty.

(18) Le fromage et les vers. L'univers d'un meunier du Xvie siècle, Paris, Flammarion 1980, traduit par Monique Aymard.

(19) J. Revel, "Micro-analyse et construction du social ", in Jeux d'échelles. La micro-analyse à l'expérience, sous la direction de J. Revel, Paris, Gallimard/Le Seuil, 1996, p. 15-36, à la p. 15. 
publié dans les Annales en 1988, souligne que " les propositions développées par la microhistoire ", " après une longue période d'attention exclusive aux processus globaux et aux structures d'ensemble, contraignent à une gymnastique intellectuelle salutaire(20) ". C'est ce même milieu historiographique et académique (I'EHESS) qui est à l'origine, en 1989, de l'édition française de L'eredità immateriale de Giovanni Levi(21), sans doute l'entreprise de traduction d'un ouvrage de la microstoria la plus complexe et ambitieuse. Dans sa longue préface du Pouvoir au village, Jacques Revel explique que "I'héritage immatériel " dont parle le livre italien - et qui n'est pas retenu dans le titre français - trouve sa traduction la plus appropriée dans la notion de "pouvoir ", un " pouvoir inédit, celui que l'on n'attendait pas, celui que l'on voit se construire tout au long du livre, c'est celui que ne garantit aucune institution et qui traduit une prise imprévue sur la réalité sociale(22)".

Phénomène circonscrit à un contexte intellectuel, la micro-histoire constitue en France un cas unique de traduction consacrée à un courant historiographique. Une exception donc, dans un marché de la traduction en sciences humaines et sociales dont Pierre Nora, une dizaine d'années plus tard, soulignait " le dramatique rétrécissement(23) ». Une exception qui a sans doute aussi valeur de symptôme des rapports complexes qui peuvent s'instaurer entre deux traditions historiographiques.

Sandro Landi (Docteur en Histoire, EUI) est historien, professeur à l'Université Bordeaux Montaigne et directeur de l'École doctorale Montaigne Humanités. Parmi ses sujets d'étude, la censure, la formation de l'opinion publique à l'époque moderne, Machiavel. Il a publié notamment Il governo delle opinioni. Censura e formazione del consenso nella Toscana del Settecento, Bologne, Il Mulino, 2000 ; Naissance de l'opinion publique dans l'Italie moderne, Rennes, Presses Universitaires de Rennes, 2006 ; Stampa, censura, opinione pubblica in età moderna, Bologne, II Mulino, 2011 ; Conoscere discosto. Saggio su Machiavelli, Bologne, Il Mulino, 2017.

(20) Annales. Économies, Sociétés, Civilisations, 43, n² 2, 1988, p. 291-293, à la p. 292.

(21) G. Levi, L'eredità immateriale. Carriera di un esorcista nel Piemonte del Seicento, Turin (Italie), Einaudi, 1985 ; traduction française par M. Aymard, Le pouvoir au village. Histoire d'un exorciste dans le Piémont du XVIIe siècle, Paris, Gallimard, 1989.

(22) J. Revel, "L'histoire au ras du sol ", in Levi, Le pouvoir au village, p. I-XXXIII, à la p. XXVIII.

(23) Pierre Nora, "Traduire : nécessité et difficultés ", Le Débat 1997/1 (n 93), p. 93-95, à la p. 94. Voir aussi, dans le même numéro de la revue Le Débat, Michel Prigent, "Sur la politique de la traduction. Entretien avec Michel Prigent ", p. 96-101. 